\title{
Diagnostic utility of linked color imaging in the evaluation of colonic mucosal inflammation in ulcerative colitis: a pilot study
}

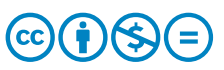

\author{
Authors \\ Nasu' ${ }^{1}$, Shinichi Hashimoto ${ }^{1}$, Michiyo Higashi ${ }^{2}$, Akio Ido \\ Institutions \\ 1 Digestive and Lifestyle Diseases, Kagoshima University \\ Graduate School of Medical and Dental Sciences, \\ Kagoshima, Japan \\ 2 Department of Pathology, Field of Oncology, Kagoshima \\ University Graduate School of Medical and Dental \\ Sciences, Kagoshima, Japan
}

Shuji Kanmura', Hitomi Hamamoto ${ }^{1}$, Akihito Tanaka1', Shiho Arima ${ }^{1}$, Fumisato Sasaki ${ }^{1}$, Shiroh Tanoue ${ }^{1}$, Yuichiro

submitted 4.7.2018

accepted after revision $\quad 8.11 .2018$

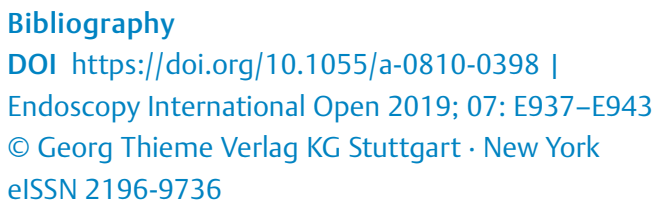

\section{Corresponding author}

Shuji Kanmura, MD PhD, Digestive and Lifestyle Diseases, Department of Human and Environmental Sciences, Kagoshima University Graduate School of Medical and Dental Sciences, 8-35-1 Sakuragaoka, Kagoshima 890-8544, Japan Fax: +81-99-2643504

skanmura@m2.kufm.kagoshima-u.ac.jp

\section{ABSTRACT}

Background and aims Recent studies recommend histological mucosal healing of intestinal tissue as a treatment goal in ulcerative colitis (UC). Linked-color imaging ( $\mathrm{LCI}$ ) is a new endoscopy system that enhances the color differences of the gastrointestinal mucosa. We investigated the efficacy of $\mathrm{LCl}$ in the evaluation of intestinal activity, including the histological activity in UC.

Method A total of 21 UC patients who were evaluated by the LASEREO system (FUJIFILM Co., Tokyo, Japan) were enrolled from August to December in 2016. All of the target points were observed by conventional white-light imaging (WLI) and $\mathrm{LCl}$ and biopsied from the region of interest in each view. We quantified the color tones of 73 biopsied points on WLI and $\mathrm{LCl}$ using the $\mathrm{L}^{*} \mathrm{a}{ }^{*} \mathrm{~b}^{*}$ color value (WLI-L, WLI-a, WLI-b, and LCI-L, LCl-a, LCl-b). We then investigated the relationships among the $L^{*} a{ }^{*} b^{*}$ color values, endoscopic findings, and histological healing.

Results The average $\mathrm{LCl}-\mathrm{a}$ and $\mathrm{LCl}-\mathrm{b}$ values of patients with high mucosal activity disease were significantly higher than in those with mucosal healing $(P<0.01)$, and only $\mathrm{LCl}$-a was significantly correlated with the score for histological healing. With regard to the other color values ( $\mathrm{LCl}-\mathrm{L}$ and all WLI values), there were no statistically significant differences in terms of average color value and correlation between patients with high mucosal activity and those with mucosal healing. In addition, the difference in the average color values with $\mathrm{LCl}$ and WLI was 7.1 and 3.1, respectively.

Conclusion $\mathrm{LCl}$ is more useful than WLI for the visualization and evaluation of mucosal inflammation in UC.

\section{Introduction}

Mucosal healing $(\mathrm{MH})$ represents an important therapeutic goal for patients with ulcerative colitis (UC), as $\mathrm{MH}$ is a predictor of clinical remission, reduced colectomy rates, and an improved quality of life [1-3]. In recent clinical trials, endoscopic $\mathrm{MH}$ was defined as a Mayo endoscopic score (MES) of 0 or 1 with conventional white-light imaging (WLI) [4-6]. However, it was reported that patients with a MES of 1 had a higher risk of re- lapse than those with a score of 0 , and the concept of $\mathrm{MH}$ should be limited to patients with a MES of 0 . MH is usually diagnosed and confirmed based on endoscopic observation using WLI [7].

It has recently been reported that histological intestinal inflammation is a valuable therapeutic target [8-12]. In the diagnosis of histological activity in UC, multiple mucosal biopsies are performed, despite the invasiveness of the procedure, because it has been reported that endoscopic and histological ac- 
tivity sometimes differ in patients with inflammatory bowel disease [13]. Thus, an endoscopic method is needed which can predict histological findings precisely with minimal invasion.

Image-enhanced endoscopy (IEE) facilitates the detection and characterization of colorectal neoplasms [14]. Recently, linked-color imaging (LCI), a color enhancement function of the LASEREO system, was developed as a new IEE system. This system enhances red and white areas making red areas appear redder, and white areas appear brighter. Thus, it is a useful tool for recognizing color differences in the mucosa, and facilitates the detection and recognition of colorectal neoplasms [15-19].

However, there are few reports on the utility of $\mathrm{LCl}$ in the assessment of histological intestinal inflammation in UC patients. If the mucosal inflammation of patients with UC could be evaluated by endoscopic observation using $\mathrm{LCl}$, without tissue biopsy, this would reduce the invasiveness of diagnostic examinations in patients with UC. The aim of the present study was to evaluate the relationship between endoscopic $\mathrm{MH}$ and histological activity in UC patients as assessed using LCI.

\section{Materials and methods}

\section{Patients' characteristics}

In total, 21 UC patients ( 8 female and 13 male; median age at diagnosis, 39.1 years) who underwent colonoscopy between August and December 2016 at Kagoshima University Hospital were enrolled in the present study. The background information on all 21 UC patients who were enrolled in the present study is summarized in $>$ Table 1 . All patients were diagnosed with UC using established endoscopic, radiological, histological, and clinical criteria. Patients with severe ulcerative colitis, infectious colitis, including Clostridium difficile infection, a history of total colectomy, or who had colorectal cancer, were excluded from the present study.

After routine bowel preparation with Moviprep that contained polyethylene glycol 3350, sodium sulfate, sodium chloride, potassium chloride, sodium ascorbate, and ascorbic acid, colonoscopy was performed by two experienced endoscopists. A total of 73 biopsied regions were analyzed in the present study ( $\triangleright$ Table 1$)$.

Each study participant underwent colonoscopy for the evaluation of treatment response, which included the documentation of endoscopic activity and $\mathrm{MH}$, or as a surveillance procedure. Mayo endoscopic scores (MESs) of 0 or 1 were defined as inactive disease and MESs of 2 or 3 as active disease [12]. The present study was approved by the Kagoshima University Hospital Institutional Review Board, and was performed in accordance with the Declaration of Helsinki. Written informed consent was obtained from all of the patients who participated in the study.

\section{Study design}

Conventional colonoscopy was performed with an EC-L600ZW endoscope with the LASEREO system that consists of VP$4450 \mathrm{HD}$ processor and LL-4450 light source (FUJIFILM Co., Tokyo, Japan), which is capable of producing light suitable for WLI and $\mathrm{LCl}$.
- Table 1 Clinical characteristics of the study patients.

Total number of patients

Sex, $M / F$

$13 / 8$

Age, years

$47.4 \pm 15.4$

Disease duration, years

$10.5 \pm 7.9$

Extent of UC

- Extensive colitis 13

- Left-sided 6

- Proctitis 3
6
2

Severity of UC

- Mild 13

- Moderate 8

- Severe 0

Clinical course

- Relapsing-remitting type 14

- Chronic continuous type 7

- One attack only 0

Treatment

- 5-Aminosalicylic acid 19

- Salazosulfapyridine 2

- Prednisolone

- Azathioprine/6-mercaptopurine

Biological therapy

2

UC, ulcerative colitis.

Each study participant underwent colonoscopy. The endoscopic images with $\mathrm{WLI}$ and $\mathrm{LCl}$ were captured from the same position in a row before the biopsy. Then the biopsy was performed targeting the reddest site in the observed area while capturing the images during the biopsy for reference of the biopsied point. In each case, the MES was evaluated under WLI observation by two experienced endoscopists.

The endoscopic images obtained by WLI and LCI before the biopsy were analyzed using Photoshop CC (Adobe Systems Inc., San Jose, California, USA). To evaluate the color value, the region of interest (ROI) was determined not only including the biopsied point but also avoiding any adverse objects such as halation, bleeding, or obvious submucosal blood vessels with a size of 50 pixels square on each image ( $\mathbf{F i g} \mathbf{1} \mathbf{1})$. The color value of the ROI was defined as the average of each pixel in the ROI using the International Commission on Illumination $1976\left(\mathrm{~L}^{*}\right.$, $a^{*}$ and $\left.b^{*}\right)$ color space. The CIE $1976\left(L^{*}, a^{*}\right.$ and $\left.b^{*}\right)$ color space (CIELAB), which is designed to approximate human perception [20], is a three-dimensional model composed of a black - white axis $\left(\mathrm{L}^{*}\right)$, a red - green axis $\left(\mathrm{a}^{*}\right)$, and a yellow - blue axis $\left(\mathrm{b}^{*}\right) . \mathrm{L}^{*}$ defines brightness, $a^{*}$ defines the red-green component, and $b^{*}$ defines the yellow - blue component. 


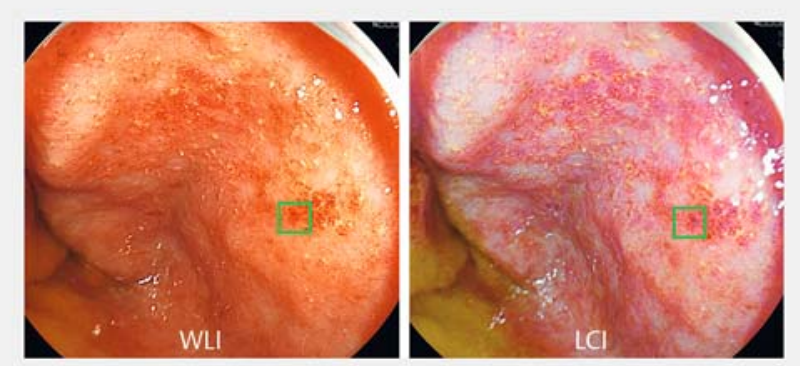

- Fig. 1 Representative case of ulcerative colitis (UC) with a Mayo endoscopic score of 1 (MES 1). The selection of sample points for the measurement of color value. Left panel: the square green area, which is the region of interest, shows a conventional image obtained by white-light imaging (WLI). Right panel: the same region was also observed by linked-color imaging (LCI).

The color difference $(\Delta E)$ between the average color of intestinal histological activity and of inactivity was calculated according to the following procedure by CIELAB: first, the averages of the $L^{*}, a^{*}$ and $b^{*}$ color values of each lesion with histological mucosal activity, defined as L1, a1, b1, and inactivity, defined as $L 2$, a2, b2, respectively, on $W L I$ and $L C I$ were calculated, then the $\Delta E$ between the lesion with histological mucosal activity and inactivity was represented using the following formula:

$\Delta E=\left\{(\mathrm{L} 1-\mathrm{L} 2)^{2}+(\mathrm{a} 1-\mathrm{a} 2)^{2}+(\mathrm{b} 1-\mathrm{b} 2)^{2}\right\}^{1 / 2}[21]$

$\triangle E$ was expressed according to the evaluation criterion of the National Bureau of Standards (NBS) units of color difference ( $\downarrow$ Table 2). $\triangle E$ was converted to NBS units using the following formula: NBS units $=\Delta E \times 0.92[22]$.

\section{Histological assessment}

The histological examinations of all biopsy specimens were performed by an experienced pathologist who was blinded to the patient's endoscopic activity. The histological assessment was based on the Geboes index [23]. The scale included six grades: 0 , structural change only; 1 , chronic inflammation; 2 , neutrophils in the lamina propria; 3, neutrophils in the epithelium; 4, crypt destruction; and 5, erosion or ulcers. A grade of 3 indicated the presence of neutrophils in the epithelium, which was representative of acute inflammation and a predictor of relapse [24]. $\mathrm{MH}$ was defined by a Geboes score $\leq 2$.

\section{Statistical analyses}

The results were analyzed using the Mann - Whitney $U$ test, or Wilcoxon signed-rank test, or Jonckheere-Terpstra trend test, as appropriate. Correlation coefficients were calculated by a Spearman's rank correlation analysis. The discriminatory power of each putative marker was described using the receiver operating characteristics area under the curve (ROC-AUC). Cutoff values were obtained from the ROC-AUC analysis. All statistical analyses were conducted using the SPSS software program (version 15, SPSS Inc., Chicago, Illinois, United States). P values $<0.05$ were considered to indicate statistical significance.
- Table 2 Evaluation criteria of color difference, based on the National Bureau of Standards (NBS) unit.

\begin{tabular}{|l|l|}
\hline NBS unit & Evaluation criterion \\
\hline $0-0.5$ & Trace \\
\hline $0.5-1.5$ & Slight \\
\hline $1.5-3.0$ & Noticeable \\
\hline $3.0-6.0$ & Appreciable \\
\hline $6.0-12.0$ & Much \\
\hline $12.0-$ & Very much \\
\hline
\end{tabular}

\section{Results}

\section{Distribution of endoscopic and pathological activity}

The distribution of endoscopic activity was as follows: MES 0 in 33 ROIs, MES 1 in 18 ROls and MES 2 in 22 ROls at the lesion. No cases of MES 3 were included, as patients with severe UC were excluded from this study. The distribution of histological activity was as follows: Geboes 1 in 7 ROls, Geboes 2 in 35 ROls, Geboes 3 in 16 ROls, Geboes 4 in 9 ROls, and Geboes 5 in 6 ROIs. The relationships between the $L^{*}, a^{*}$ and $b^{*}$ color values on WLI or $\mathrm{LCl}$ and the Geboes score were investigated. The WLI-L, WLIa, WLI-b and LCI-L, LCl-a, LCl-b values were indicated as $\mathrm{L}^{*}$, a* and $b^{*}$ color values on $\mathrm{WLI}$ and $\mathrm{LCl}$, respectively. The Jonckheere-Terpstra trend test showed that the color values of $\mathrm{LCl}-\mathrm{a}, \mathrm{LCl}-\mathrm{b}$, and WLI-b tended to be significantly higher when the Geboes score was also high $(P=0.003,0.03$, and 0.01 , respectively) ( $\mathbf{F i g . 2}$ ). No significant differences were observed between the Geboes score and the WLI-L, WLI-a, or LCI$\mathrm{L}$ color values. Furthermore, only the $\mathrm{LCl}$-a color value was significantly correlated with the Geboes score using Spearman's rank correlation analysis $(r=0.36, P<0.01)$. No significant differences were observed between the Geboes score and the WLI-L, WLI-a, WLI-b, LCI-L, or LCI-b color values using Spearman's rank correlation analysis.

\section{Comparison of the $L^{*}, a^{*}$ and $b^{*}$ color values of $W L I$ or $\mathrm{LCl}$ in patients with $\mathrm{UC}$}

The average WLI-L, WLI-a, WLI-b, LCI-L, LCl-a, and LCI-b color values in UC patients with mucosal activity were 52.4, 40.6, $40.9,57.5,29.1$, and 23.4, respectively. The average WLI-L, WLI-a, WLI-b, LCI-L, LCI-a, and LCI-b color values in UC patients with $\mathrm{MH}$ were $52.5,38.7,38.2,56.9,23.0$, and 18.8 , respectively. The differences in the $\mathrm{L}^{*}, \mathrm{a}^{*}$ and $\mathrm{b}^{*}$ color values on $\mathrm{WLI}$ or $\mathrm{LCl}$ according to mucosal inflammation were analyzed. The average $\mathrm{LCl}-\mathrm{a}$ and $\mathrm{LCl}-\mathrm{b}$ values of patients with high mucosal activity were statistically significantly higher than those of patients with $\mathrm{MH}$, while there was no significant difference in the other color values between patients with high mucosal activity and those with $\mathrm{MH}$ ( $\triangleright$ Fig. 3 ).

The color difference $(\Delta E)$ between the average color of intestinal histological activity and of inactivity was 3.1 on WLI and 7.1 on $\mathrm{LCl}$, which was a statistically significant difference $(P<$ 

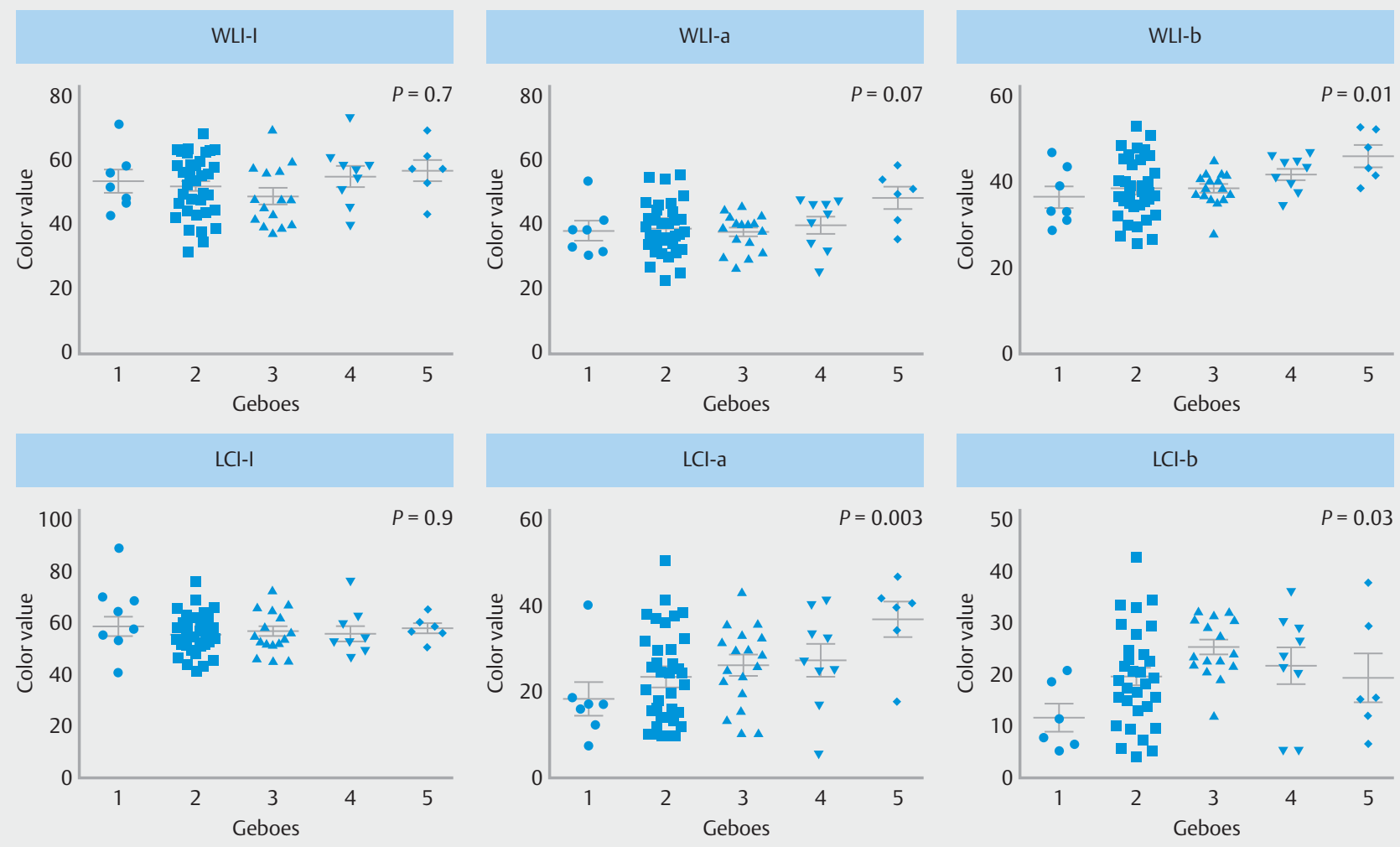

$N=73$

- Fig. 2 Distribution of the $L^{*}, a^{*}$ and $b^{*}$ color values obtained by white-light imaging (WLI) and linked-color imaging (LCI) for each pathological activity (Geboes score). The color values of LCl-a, LCI-b, and WLI-b tended to be significantly higher, when the Geboes score was also high. Each symbol represents one patient. (Jonckheere-Terpstra trend test)

0.05). The assessment of the color difference-based NBS unit indicated that it was "much" with $\mathrm{LCl}$, whereas it was "appreciable" with WLI ( $\triangleright$ Table 2 ).

\section{Diagnostic utility of the $L^{*}, a^{*}$ and $b^{*}$ color values on WLI or $\mathrm{LCl}$ and the Geboes score of intestinal histological activity}

The WLI-a, WLI-b, LCl-a, and LCI-b color values were investigated to evaluate their utility in distinguishing between histological mucosal activity and inactivity. The ROC-AUCs for WLI-a and WLI-b did not differ to a statistically significant extent $(P=0.27$ and $P=0.06$, respectively). In contrast, the ROC-AUCs for $\mathrm{LCl}-\mathrm{a}$ and LCl-b were 0.67 and 0.66 , respectively $(P=0.01$ and $P=$ 0.02 ) ( Fig.4). The ROC-AUC analysis revealed that an LCl-a of 23.2 and an LCl-b of 19.9 were the optimum cutoff values for discriminating between the histologically active mucosa and histological MH (sensitivity, 74.2\%; specificity, $57.1 \%$; positive predictive value, $56.1 \%$; negative predictive value, $75 \%$; accuracy, 64.4\%; LCl-a and LCI-b had exactly the same values). In addition, the ROC-AUC analysis revealed that an WLI-a of 38.8 and an WLI-b of 38.5 were the optimum cutoff values for discriminating between the histologically active mucosa and histological $\mathrm{MH}$. The sensitivity of WLI-a and WLI-b were 64.5 $\%$ and $71.0 \%$; specificity, $57.1 \%$ and $54.8 \%$; positive predictive value, $52.6 \%$ and $53.7 \%$; negative predictive value, $68.6 \%$ and $71.9 \%$; accuracy, $60.2 \%$ and $61.7 \%$, respectively ( $\triangleright$ Table 3 ).

\section{Discussion}

This is the first study to investigate the correlation between the color values detected by the $\mathrm{L}^{*}, \mathrm{a}^{*}$ and $\mathrm{b}^{*}$ color values on WLI and $\mathrm{LCl}$ and histological assessment based on the Geboes index in patients with UC. The findings of endoscopic observations by $\mathrm{LCl}$-a and $\mathrm{LCl}-\mathrm{b}$ showed a significant difference between mucosal inflammation and non-inflammation, and LCl-a was significantly correlated with the histological mucosal inflammation score. The color difference between inflammation and non-inflammation was more than twice the average difference for $\mathrm{LCl}$ compared with WLI. Therefore, $\mathrm{LCl}$ would be more useful than WLI for inflammatory diagnosis of UC.

The endoscopic color values observed by LCI were correlated with microscopic/histological mucosal activity. We evaluated the color difference between pathological inflammation and remission. Since microscopic/histological mucosal activity is reported to be a predictor of relapse in UC patients [8-12], the endoscopic color difference on LCl may be used to diagnose mucosal activity in addition to a biopsy. 

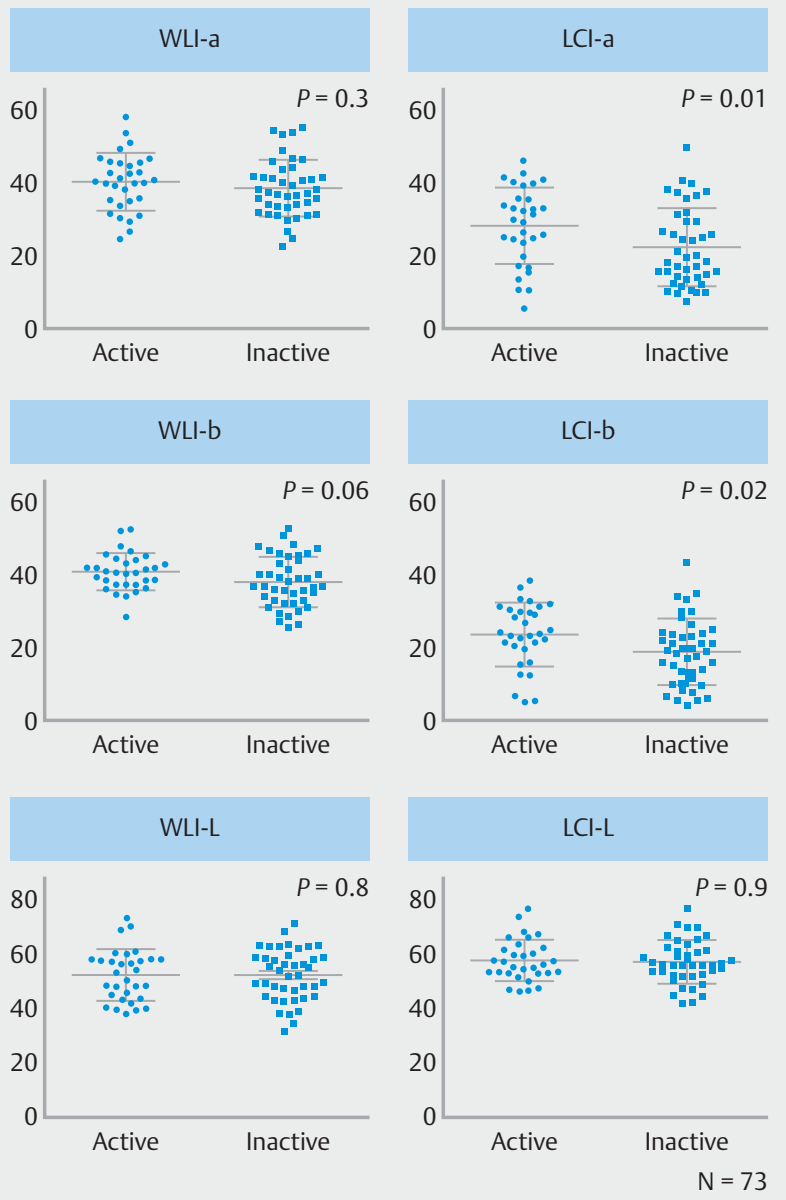

- Fig. 3 Comparison of the $\mathrm{L}^{*}, \mathrm{a}^{*}$ and $\mathrm{b}^{*}$ color values obtained by white-light imaging (WLI) and linked-color imaging ( $\mathrm{LCI})$ in patients with histologically inactive ulcerative colitis (UC) versus those with histologically active UC. The $L^{*}, a^{*}$ and $b^{*}$ color values of UC patients with histologically active disease were higher than those of patients with histologically inactive disease. In particular, the median LCl-a and LCl-b levels in patients with histologically active UC were significantly higher than those in patients with histologically inactive UC. Each symbol represents one patient. (MannWhitney U test)

Recent clinical trials of endoscopic criteria have used the Mayo endoscopic score (MES), which is easy to apply in clinical trials and clinical practice. The Mayo endoscopic scores are defined as follows: MES 0, normal mucosa or inactive disease; MES 1, mild activity (erythema, decreased vascular pattern, mild friability); MES 2, moderate activity (marked erythema, lack of vascular pattern, friability, erosions); MES 3, severe activity (spontaneous bleeding, large ulcerations). Conventional endoscopy with WLI is normally performed to evaluate intestinal activity in UC. However, the findings of conventional endoscopy using WLI do not always correlate with the histological findings $[25,26]$. Indeed, in this study, $28 \%$ of patients with MES 1 showed histological activity (data not shown) (> Fig.4). Thus, the MES, as determined by WLI, was insufficient for the diagnosis of histological activity, especially with regard to the

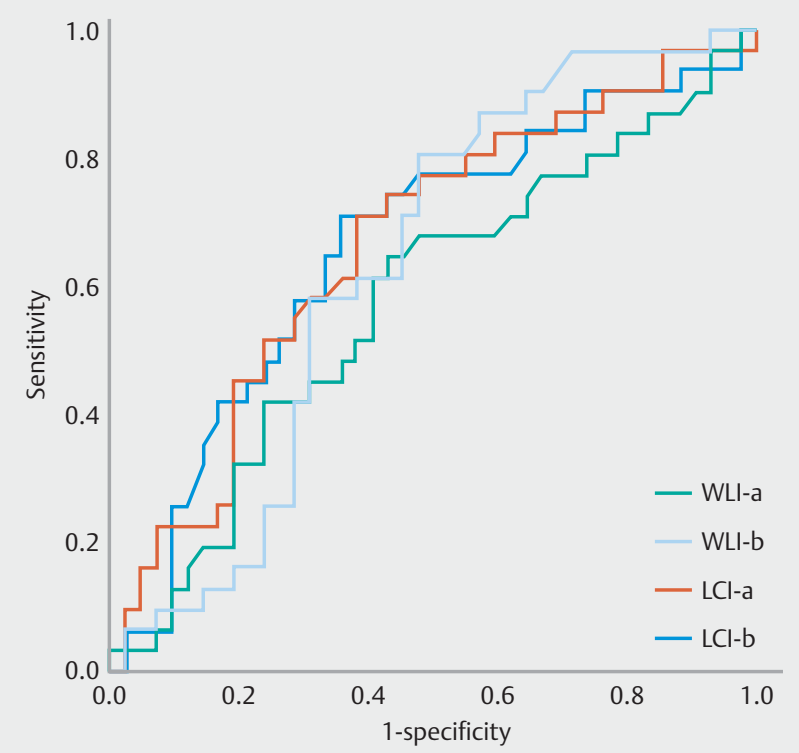

- Fig. 4 The receiver operating characteristic (ROC) curves of WLI-a, WLI-b, LCl-a, and LCI-b for the diagnosis of mucosal healing of ulcerative colitis (UC). The ROC curves for each white-light imaging (WLI) and linked-color imaging ( $\mathrm{LCl}$ ) color value were obtained by plotting sensitivity versus 1 - specificity. The ROC areas under the curve for WLI-a, WLI-b, LCI-a, and LCI-b were $0.58,0.63$, 0.67 , and 0.66 , respectively $(P=0.3,0.06,0.01$, and 0.02 , respectively).

diagnosis of mild inflammatory activity [12]. LCl observation can emphasize redness (i.e., reddish areas indicate erythema), the color difference presumably increasing between active and nonactive lesions, and it is easi to recognize lesions showing erythema. We therefore focused on the relationship between the colors observed by endoscopy using $\mathrm{LCl}$ and tissue inflammation.

Uchiyama et al. recently reported that $\mathrm{LCl}$ could be used both to evaluate colonic mucosal inflammation and predict the outcome in UC patients. They classified endoscopic findings on $\mathrm{LCl}$ based on redness with or without visible vessels and reported that the endoscopic $\mathrm{LCl}$ classification was correlated with the $\mathrm{LCl}$ index [27]. We also investigated the color values of the images using CIELAB, which is designed to approximate human perception [20]. It was reported that the color of mucosa on WLI and $\mathrm{LCl}$ consists of two dimensions between a positive $a^{*}$ value, indicated in red, and a positive $b^{*}$ value, indicated in yellow [21]. The observation of $\mathrm{LCl}-\mathrm{a}$ and $\mathrm{LCl}-\mathrm{b}$ emphasized the redness and yellowness of the lesion and highlighted the areas of inflammation in this study. The color difference, including $\mathrm{LCl}-\mathrm{a}$ and $\mathrm{LCl}-\mathrm{b}$, under $\mathrm{LCl}$ observation was significantly higher than that under WLI. We therefore demonstrated that $\mathrm{LCl}$ observation was useful for detecting and visualizing mucosal inflammation.

Previous studies have reported the utility of endoscopic observation using $\mathrm{LCl}$ in screening for gastrointestinal tumors, as this approach increases the discriminatory power of the red color tone [22, $28-30]$. In the present study, $\mathrm{LCl}$ was also able to 
- Table 3 Receiver operating characteristics analysis for the diagnosis of pathological mucosal healing in ulcerative colitis.

\begin{tabular}{|c|l|l|l|l|l|l|l|}
\hline & ROC-AUC & Sensitivity, $\%$ & Specificity, $\%$ & PPV, $\%$ & NPV, $\%$ & Accuracy, \% \\
\hline WLI-a & 0.58 & 64.5 & 57.1 & 52.6 & 68.6 & 60.2 & 0.3 \\
\hline WLI-b & 0.63 & 71.0 & 54.8 & 53.7 & 71.9 & 61.7 & 0.06 \\
\hline LCl-a & 0.67 & 74.2 & 57.1 & 56.1 & 75 & 64.4 & 0.01 \\
\hline LCl-b & 0.66 & 74.2 & 57.1 & 56.1 & 75 & 64.4 & 0.02 \\
\hline
\end{tabular}

WLI, white-light image; LCI, linked-color image; ROC-AUC, receiver operating characteristic area under the curve; PPV, positive predictive value; NPV, negative predictive value.

improve the visibility of inflammation in the red region of the colonic mucosa in patients with UC and provided brighter images, allowing for both the lesion and the background to be visualized more clearly than with other IEE systems such as narrow-band imaging or blue laser imaging, in which the background appears dark. For these reasons, $\mathrm{LCl}$ is suitable for use in screening for mucosal inflammation.

Many histological scores for assessing disease activity in UC have been described since the 1950 s, although none have been fully validated. The Geboes score is commonly used as a histological index of disease activity in UC. This index assesses the following features: architectural change, lamina propria neutrophils and eosinophils, neutrophils in epithelium, crypt destruction, and erosion or ulceration. Given that a previous study reported this score to be the best validated of all available scores [31], we evaluated the mucosal inflammation of biopsied tissue using the Geboes score.

We showed that the endoscopic color value on $\mathrm{LCl}$ was correlated with microscopic/histological mucosal activity; as such, $\mathrm{LCl}$ would be useful not only for the detection of inflammation but also for the characterization of mucosal inflammation. However, the color value of the ROI was only calculated after colonoscopy for clinical application. It would be more useful if the system could immediately show the color values of the ROI to predict mucosal inflammation during colonoscopy. Furthermore, evaluation of the ROI was only performed for a single point; another tool able to analyze the color value of a large area is therefore needed. In addition, since the accuracy of the $\mathrm{LCl}$-a diagnosis of mucosal inflammation at the ROI only showed mild correlation with the pathological diagnosis, the $\mathrm{LCl}$ diagnosis cannot completely replace pathological diagnosis at present. Therefore, a new classification system using $\mathrm{LCl}$ is needed as an alternative to existing classification systems using conventional WLI.

Only the color value examination findings by WLI or $\mathrm{LCl}$ showed a low diagnosis rate. We have found that, even in patients without UC, the color value of the colonic mucosa-based endoscopic findings showed individual variations regardless of whether mucosal inflammation was present or not. As a result, individual differences in mucosal color was one of the factors giving increased color values. Therefore, there are limitations when attempting to diagnose mucosal inflammation based on the absolute color values alone. However, since a correlation was observed between the color value of $\mathrm{LCl}$-a and the degree of pathological inflammation, the color value is therefore considered to be an indicator for comprehensively distinguishing mucosal inflammation. The color value by $\mathrm{LCl}$ was significantly correlated with mucosal inflammation compared to WLI. It would also be useful if such a system based on the combination of color values by $\mathrm{LCl}$ and other parameters, such as any visible vessels, or ulceration lesions similar to the Mayo classification, could diagnose mucosal activity with high accuracy. It therefore appears to be promising to compare the findings of $\mathrm{LCl}$ observations with those of conventional WLI observations.

The present study has several limitations. Although this was a prospective study, it was performed at a single center as a pilot study, and the population was relatively small. It will be difficult to perform evaluations based on the $L^{*}, a^{*}$ and $b^{*}$ color values in cases with ulcerative lesions that may be coated with purulent mucus or have inflammatory polyps. In addition, the $L^{*}, a^{*}$ and $b^{*}$ color values changed slightly depending on the observation distance. The color value of the ROI could not be obtained quickly during endoscopic examination; thus, a system must be established in the future to obtain the color value of the ROI. Finally, because the ROI was a tiny spot, the color value was affected by the color of small structures, such as capillaries at the mucosal surface and partially hidden vessels in the submucosal layer; we should therefore investigate the method to decide the suitable ROI which is able to estimate mucosal inflammation most efficiently.

In conclusion, we investigated the relationship between histological activity and color value using $\mathrm{LCl}$ and WLI. The $\mathrm{LCl}-\mathrm{a}$ and LCl-b color values were useful parameters for diagnosing histological mucosal activity, and LCl observation was useful for the visualization and evaluation of mucosal inflammation in UC.

\section{Acknowledgments}

We thank Dr. Yuichi Teramura, FUJIFILM for his valuable technical advice.

\section{Competing interests}

None 
[1] Frøslie KF, Jahnsen J, Moum BA et al. Mucosal healing in inflammatory bowel disease: results from a Norwegian population-based cohort. Gastroenterology 2007; 133: 412-422

[2] Colombel JF, Rutgeerts P, Reinisch W et al. Early mucosal healing with infliximab is associated with improved long-term clinical outcomes in ulcerative colitis. Gastroenterology 2011; 141: 1194-1201

[3] Casellas F, Barreiro de Acosta M, Iglesias M et al. Mucosal healing restores normal health and quality of life in patients with inflammatory bowel disease. Eur J Gastroenterol Hepatol 2012; 24: 762 - 769

[4] Schroeder KW, Tremaine WJ, Ilstrup DM. Coated oral 5-aminosalicylic acid therapy for mildly to moderately active ulcerative colitis. A randomized study. NEJM 1987; 317: 1625-1629

[5] Rutgeerts P, Sandborn W], Feagan BG et al. Infliximab for induction and maintenance therapy for ulcerative colitis. NEJM 2005; 353 : $2462-2476$

[6] Sandborn WJ, Feagan BG, Marano C et al. Subcutaneous golimumab induces clinical response and remission in patients with moderate-tosevere ulcerative colitis. Gastroenterology 2014; 146: 85 - 95

[7] Barreiro-de Acosta M, Vallejo N, de la Iglesia D et al. Evaluation of the risk of relapse in ulcerative colitis according to the degree of mucosal healing (Mayo 0 vs 1): A longitudinal cohort study. J Crohns Colitis 2015; 10: $13-19$

[8] D’Haens G, Sandborn WJ, Feagan BG et al. A review of activity indices and efficacy end points for clinical trials of medical therapy in adults with ulcerative colitis. Gastroenterology 2007; 132: $763-786$

[9] Hanauer SB, Kirsner JB. Treat the patient or treat the disease? Dig Dis 2012; 30: 400-403

[10] Riley SA, Mani V, Goodman MJ et al. Microscopic activity in ulcerative colitis: what does it mean? Gut 1991; 32: $174-178$

[11] Bitton A, Peppercorn MA, Antonioli DA et al. Clinical, biological, and histologic parameters as predictors of relapse in ulcerative colitis. Gastroenterology 2001; 120: 13-20

[12] Neurath MF, Travis SP. Mucosal healing in inflammatory bowel diseases: a systematic review. Gut 2012; 61: 1619-1635

[13] Itzkowitz SH, Present DH. Consensus conference: Colorectal cancer screening and surveillance in inflammatory bowel disease. Inflamm Bowel Dis 2005; 11: 314-321

[14] Matsuda T, Ono A, Sekiguchi M et al. Advances in image enhancement in colonoscopy for detection of adenomas. Nat Rev Gastroenterol Hepatol 2017; 14: 305-314

[15] Okada M, Sakamoto H, Takezawa T et al. Laterally spreading tumor of the rectum delineated with linked color imaging technology. Clin Endosc 2016; 49: $207-208$

[16] Yoshida N, Naito Y, Yasuda R et al. Linked color imaging improves the visibility of various featured colorectal polyps in an endoscopist's vis-

ibility and color difference value. Int I Colorectal Dis 2017; 32: 1253 1260

[17] Yoshida N, Naito Y, Murakami T et al. Linked color imaging improves the visibility of colorectal polyps: a video study. Endosc Int Open 2017; 5: E518-E525

[18] Suzuki T, Hara T, Kitagawa Y et al. Linked-color imaging improves endoscopic visibility of colorectal nongranular flat lesions. Gastrointest Endosc 2017; 86: 692 - 697

[19] Fujimoto D, Muguruma N, Okamoto $\mathrm{K}$ et al. Linked color imaging enhances endoscopic detection of sessile serrated adenoma/polyps. Endosc Int Open 2018; 6: E322 - E334

[20] Kuehni RG. Color-tolerance data and the tentative CIE $1976 \mathrm{~L}$ a b formula. J Opt Soc Am 1976; 66: 497-500

[21] Mizukami K, Ogawa R, Okamoto K et al. Objective endoscopic analysis with linked color imaging regarding gastric mucosal atrophy: a pilot study. Gastroenterol Res Pract 2017; 2017: 5054237

[22] Kanzaki H, Takenaka R, Kawahara Y et al. Linked color imaging (LCI), a novel image-enhanced endoscopy technology, emphasizes the color of early gastric cancer. Endosc Int Open 2017; 5: E1005-E1013

[23] Geboes K, Riddell R, Ost A et al. A reproducible grading scale for histological assessment of inflammation in ulcerative colitis. Gut 2000; 47: $404-409$

[24] Li CQ, Xie XJ, Yu T et al. Classification of inflammation activity in ulcerative colitis by confocal laser endomicroscopy. Am J Gastroenterol 2010; 105: 1391 - 1396

[25] Bryant RV, Burger DC, Delo J et al. Beyond endoscopic mucosal healing in UC: histological remission better predicts corticosteroid use and hospitalisation over 6 years of follow-up. Gut 2016; 65: 408-414

[26] Zenlea T, Yee EU, Rosenberg L et al. Histology grade is independently associated with relapse risk in patients with ulcerative colitis in clinical remission: a prospective study. Am J Gastroenterol 2016; 111: 685 690

[27] Uchiyama K, Takagi T, Kashiwagi S et al. Assessment of endoscopic mucosal healing of ulcerative colitis using linked colour imaging, a novel endoscopic enhancement system. J Crohns Colitis 2017; 11: $963-969$

[28] Yoshifuku Y, Sanomura Y, Oka S et al. Evaluation of the visibility of early gastric cancer using linked color imaging and blue laser imaging. BMC Gastroenterol 2017; 17: 150

[29] Sun X, Bi Y, Dong T et al. Linked colour imaging benefits the endoscopic diagnosis of distal gastric diseases. Sci Rep 2017; 7: 5638

[30] Fukuda H, Miura Y, Hayashi Y et al. Linked color imaging technology facilitates early detection of flat gastric cancers. Clin J Gastroenterol 2015; 8: 385-389

[31] Bryant RV, Winer S, Travis SP et al. Systematic review: Histological remission in inflammatory bowel disease. Is 'complete' remission the new treatment paradigm? An IOIBD initiative. J Crohns Colitis 2014; 8: $1582-1597$ 